\title{
Auswirkung epidemiologischer \\ Daten, anamnestischer \\ Angaben und klinischer \\ Untersuchungsbefunde auf \\ die Hörentwicklung nach \\ explorativer Tympanoskopie \\ mit Abdichtung der runden/ \\ ovalen Fensternische bei \\ Hörsturzpatienten
}

\subsection{Einleitung}

\subsubsection{Hinführung zum Thema}

Viele Patienten interessiert recht bald, nachdem ihre Diagnose - in diesem Fall „Hörsturz“ - gestellt worden ist, mit welchen prognostischen Erwartungen sie bezüglich ihrer Hörerholung rechnen können. Der seriös argumentierende Arzt wird antworten, dass dies von einigen Ausgangsbedingungen, nicht zuletzt aber auch von dem auszuwählenden Therapieverfahren abhängig ist, unterscheidet sich also gewöhnlich von angewendetem Behandlungs- zu Behandlungsverfahren. Insofern ist man im Vergleich der in der eigenen Studiengruppe erhobenen Ergebnisse mit denen, die aus der Literatur zusammengetragen werden, darauf angewiesen, sich auf die Auswahl der Publikationen zu beschränken, bei denen nicht nur gleichartige therapeutische Vorgehensweisen gewählt wurden, sondern auch die sonstigen Rahmenbedingungen des Studienprotokolls weitgehend identisch waren. Dies trifft für die Tympanoskopie mit Abdeckung der runden/ ovalen Fensternische mit körpereigenem Bindegewebe, das nicht mit einem Kortikoid getränkt wurde, nur für einen Teil der Publikationen zu [29, 30, 33, 57, 65, $75,78,97,101]$.

\subsubsection{Fragestellung}

In diesem Kapitel soll auf Zusammenhänge eingegangen werden, die sich aus verschiedenen epidemiologischen Daten, anamnestischen Angaben sowie ärztlich erhobenen Erstbefunden nach der Krankenhausaufnahme und der weiteren 
Entwicklung des Hörgeschehens nach der Tympanoskopie ergaben. In die Auswertung konnten 42 Patienten des eigenen Krankengutes und 585 Patienten aus 8 Publikationen einbezogen werden (Tab. 1.2) [29, 30, 33, 57, 75, 78, 97, 101].

\subsection{Ausgangslage}

\subsubsection{Rekrutierung des Patientengutes}

Von den 42 Patienten des eigenen Krankengutes wurde ein mittlerer Hörgewinn von 28,2 dB (SAW: 26,8 dB) erreicht (Abb. 5.1). Damit hatte sich die Hörleistung, ausgehend von einem initialen Hörverlust von 101,0 dB (SAW: 16,6 dB), auf einen durchschnittlicher Endhörverlust von 72,8 dB (SAW: 31,8 dB) verbessert. Abb. 5.1 zeigt, dass sich die Hörkurve im Tiefton- und Mitteltonbereich fast um das Doppelte besser erholt hatte als dies im Hochfrequenzbereich der Fall war (Mittelwert Hörgewinn bei 0,25 und 0,5 kHz: 44,8 dB, Mittelwert Hörgewinn bei 6 und $8 \mathrm{kHz}: 22,6 \mathrm{~dB}$ ).

\subsection{Ergebnisse}

Nachfolgend werden nur die Auswertungsergebnisse aufgelistet, bei denen durch Unterteilung des Gesamtpatientengutes in zwei Subpopulationen - je nachdem, ob ein Merkmalskriterium erfüllt oder nicht erfüllt war - im statistischen Vergleich der Hörgewinne und Endhörverluste signifikante Unterschiede $(\mathrm{p}<0,05)$ ermittelt werden konnten (Tab. 5.1).

\subsubsection{Beziehung zwischen Anfangs- zu Endhörverlusten}

Zunächst konnte festgestellt werden, dass in unserem Patientengut die gemittelten Endhörverluste hochsignifikant von den Ausgangshörverlusten differierten ( $\mathrm{p}=$ 0,014 nach Bonferroni-Korrektur und $\mathrm{p}=0,006$ nach Li-Korrektur) (Tab. 5.1 und Abb. 5.1).

Mit dieser Beobachtung standen wir in Übereinstimmung mit den Ergebnissen anderer Autoren, bei denen sich die im Rahmen der Nachuntersuchung dokumentierten Hörverluste ebenfalls jeweils hochsignifikant von den mittleren Tonhörverlusten zu Beginn des Klinikaufenthaltes unterschieden hatten (Tab. 5.2) [29, 75, 78, 97]. Diese Feststellung sollte, wie bereits früher erwähnt, jedoch nicht zu der automatischen Interpretation verleiten, allein der Tympanoskopie diesen Erfolg dem Hörgewinn zuzuschreiben. 


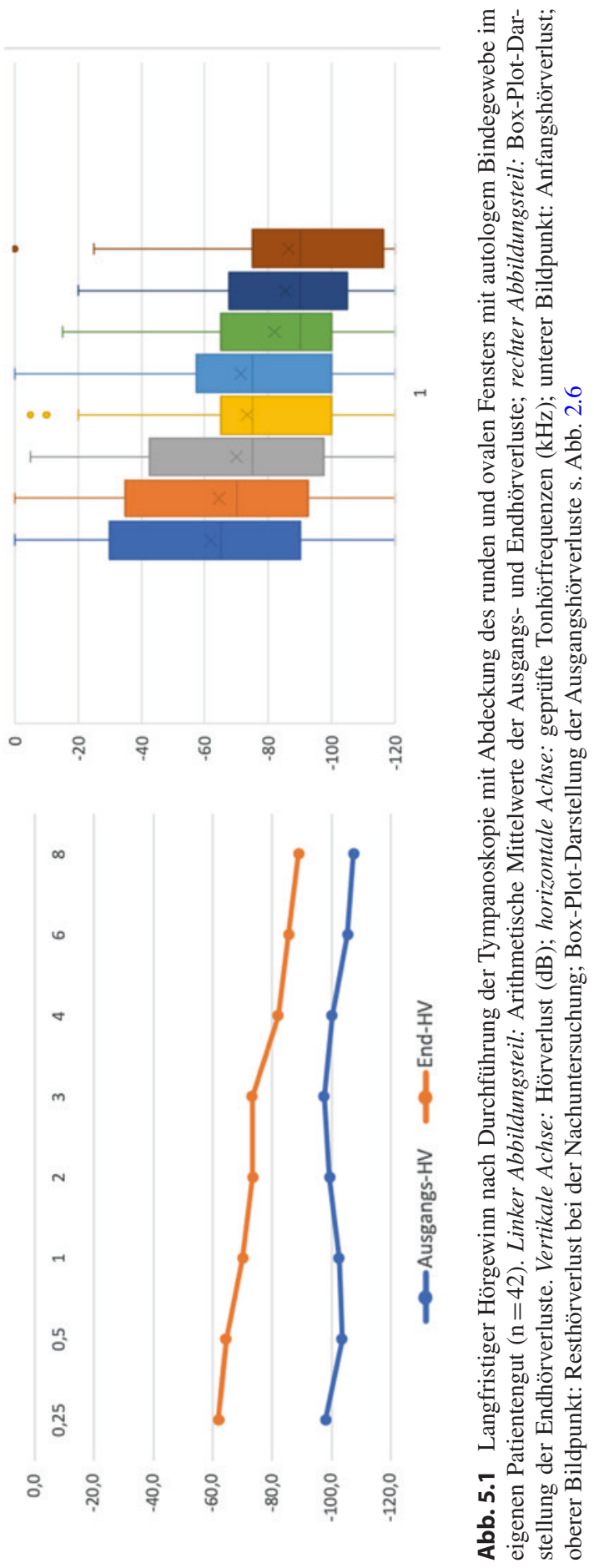


Tab. 5.1 Darstellung statistisch signifikanter und nichtsignifikanter Beziehungen zwischen dem Zutreffen bzw. Nichtzutreffen gesetzter Filterkriterien bei den epidemiologischen Daten, anamnestischen Angaben und ärztlich erhobenen Erstbefunde bei Krankenhausaufnahme zum mittleren Hörgewinn und Endhörverlust im eigenen Krankengut $(n=42)$

\begin{tabular}{|c|c|c|c|c|c|c|}
\hline & Hörgewinn & $\begin{array}{l}\text { Nach } \\
\text { Bonferroni- } \\
\text { Korrektur }\end{array}$ & $\begin{array}{l}\text { Nach Li- } \\
\text { Korrektur }\end{array}$ & End-HV & $\begin{array}{l}\text { Nach } \\
\text { Bonferroni- } \\
\text { Korrektur }\end{array}$ & $\begin{array}{l}\text { Nach Li- } \\
\text { Korrektur }\end{array}$ \\
\hline Geschlecht & 0,38 & & & 0,23 & & \\
\hline Alter & 0,68 & & & 0,75 & & \\
\hline Seite & 0,09 & & & 0,72 & & \\
\hline $\begin{array}{l}\text { Vor- } \\
\text { erkrankung }\end{array}$ & $0,028^{*}$ & 0,39 & 0,48 & 0,27 & & \\
\hline Vortherapie & 0,24 & & & 0,70 & & \\
\hline Auslöser & 0,87 & & & $0,036^{*}$ & 0,504 & 0,07 \\
\hline Schwindel & 0,15 & & & $0,003^{*}$ & $0,042^{*}$ & $0,02^{*}$ \\
\hline SPN & 0,06 & & & $0,013^{*}$ & 0,182 & \\
\hline Tinnitus & 0,78 & & & 0,75 & & \\
\hline IOP & 0,18 & & & 0,21 & & \\
\hline $\begin{array}{l}\mathrm{HS} \rightarrow \text { Stat. } \\
\mathrm{A}\end{array}$ & 0,30 & & & 0,22 & & \\
\hline Stat & 0,32 & & & 0,16 & & \\
\hline Gesamtzeit & 0,97 & & & 0,82 & & \\
\hline $\begin{array}{l}\text { Ausgangs- } \\
\text { HV }\end{array}$ & 0,58 & & & $0,001^{* *}$ & $0,014^{*}$ & $0,006^{*}$ \\
\hline
\end{tabular}

$S P N=$ Spontannystagmus; $I O P=$ intraoperativ pathologischer Befund; $H S \rightarrow$ Stat. $A=$ Latenzzeit zwischen Hörsturzereignis und stationärer Krankenhausaufnahme; Stat $=$ Stationäre Behandlungszeit bis zum Tympanoskopietermin; Gesamtzeit=Latenzzeit zwischen Hörsturzereignis und Tympanoskopietermin; Ausgangs- $H V=$ Anfangshörverlust; "signifikanter Unterschied; **hochsignifikanter Unterschied

\subsubsection{Frequenzbezug der Hörerholung}

Die Tatsache, dass das Erholungsvermögen von den tiefen zu den hohen Frequenzen hin abnimmt, erklärt sich möglicherweise partiell dadurch, dass es sich mit einem Durchschnittsalter von 65 Jahren um ein vorwiegend älteres Patientenkollektiv handelt, bei dem schon vor dem Hörsturzereignis im Hochtonbereich fortgeschrittene Hörverluste bestanden haben mögen. Dementsprechend würden die Hörgewinne in diesem Frequenzbereich auch keine so ausgeprägten Verbesserungen erwarten lassen, wie es bei jüngeren Erkrankten der Fall wäre. Eine vergleichbare Beobachtungen fand sich auch in den Veröffentlichungen von Habner et al., Kampfner et al., Maier et al., Reineke et al. und Thomas et al. [29, $33,57,78,97]$. 


\subsubsection{Epidemiologische Daten und Hörerholung}

Die Geschlechtszugehörigkeit, das Alter des Patienten, die Seitenlokalisation des Hörsturzes, eine Vorerkrankung am Innenohr oder früheres SHT oder die Tatsache, ob ambulant vor dem Krankenhausaufenthalt eine orale Behandlung durchgeführt worden war, hatte bei unseren Patienten keinen statistisch signifikanten Einfluss auf die Hörentwicklung nach explorativer Tympanoskopie mit Abdeckung beider Fensternischen (Tab. 5.1).

Weder das Geschlecht der aus der Literatur zusammengetragenen Untersuchungsgruppen noch die Seitenlokalisation der Hörminderung waren signifikant beeinflussend auf den Hörgewinn und die Höhe des Endhörverlustes (Tab. 5.2).

Die Frage, ob sich das Alter der Patienten auf die weitere Entwicklung des Hörvermögens nach der Tympanoskopie auswirkt, wird kontrovers diskutiert. Kampfner et al. hatte eine Abhängigkeit des Hörgewinns nach Hörsturz vom Alter der Patienten festgestellt (bessere Hörerholung bei jüngeren Patienten), Thomas et al. fanden einen statistischen Zusammenhang zwischen dem Alter und dem postoperativ langfristig gemessenen Hörvermögen (Tab. 5.2) [33, 97]. Alle anderen Autoren, die der Frage nachgegangen waren, ob sich der langfristig verbliebene Hörverlust zwischen den beiden Subpopulationen der älteren und jüngeren Patienten unterscheidet, konnten keinen entsprechenden Zusammenhang in ihrem Krankengut finden [29, 78]. Wir konnten eine derartige Assoziation des Alters mit dem Regenerationsvermögen des Hörorganes ebenfalls nicht bestätigen. Einen eindeutigen Prognosewert für das Hörerholungsvermögen scheint das Alter der betroffenen Patienten also nicht darzustellen. Dieses Argument kann insbesondere bei der Aufklärung älterer Patienten in dem Sinne hilfreich sein, dass auch ihnen durch eine vorgesehene explorative Tympanoskopie noch gute Chancen für eine Hörverbesserung in Aussicht gestellt werden kann.

\subsubsection{Innenohrvorerkrankung/Schädel-Hirn-Trauma und Hörerholung}

Lagen vor dem Hörsturz bereits eine Vorerkrankung am Innenohr oder ein früheres SchädelHirn-Trauma vor, bestanden in unserem Krankengut in einem ersten Auswertungsschritt signifikante Unterschiede bezüglich der Höhe des Hörgewinns im Vergleich zu Patienten ohne Vorerkrankungen $(p=0,028$; Hörgewinn mit Vorerkrankungen: 9,8 dB, SAW: 30,9 dB, $n=11$; Hörgewinn ohne Vorerkrankungen: $34,7 \mathrm{~dB}$, SAW: $22,7 \mathrm{~dB}, \mathrm{n}=30$ ). Nach der Bonferroniund auch Li-Korrektur ließen sich diese signifikanten Unterschiede jedoch nicht mehr bestätigen (nach Bonferroni-Korrektur $\mathrm{p}=0,392$; nach Li-Korrektur $\mathrm{p}=0,48)(\mathrm{Tab} .5 .1)$.

Der Frage, ob sich Innenohr-bedingte Vorerkrankungen auf die Hörmesswerte auswirken, waren auch Thomas et al. nachgegangen (Tab. 5.2) [97]. Diese Autorengruppe fand ebenfalls keine signifikanten Auswirkungen auf den Hörgewinn und die Hördaten. Immerhin wäre es auf den ersten Blick durchaus plausibel, dass sich 
Tab. 5.2 Darstellung statistisch signifikanter und nicht signifikanter Beziehungen zwischen dem Vorhandensein bzw. Nichtvorhandensein bestimmter Filterkriterien bei einzelnen epidemiologischen Daten, anamnestischen Angaben und ärztlich erhobenen Erstbefunden bei der Krankenhausaufnahme zum präoperativen Hörverlust und dem mittleren Endhörverlust

\begin{tabular}{|c|c|c|c|c|c|c|}
\hline Studie & & Geschlecht & Alter & Seite & Vorerkrankung & Vorther \\
\hline \multirow{2}{*}{$\begin{array}{l}\text { Haubner } \\
\text { et al. (29) }\end{array}$} & Post-OP-HV & ns & ns & & & \\
\hline & $\mathrm{HG}$ & & & & & \\
\hline \multirow{2}{*}{$\begin{array}{l}\text { Hoch et al. } \\
(30)\end{array}$} & Post-OP HV & & & & & \\
\hline & HG & & & & & \\
\hline \multirow{2}{*}{$\begin{array}{l}\text { Kampfner } \\
\text { et al. (33) }\end{array}$} & Post-OP-HV & & & & & \\
\hline & $\mathrm{HG}$ & ns & sssssss & & & \\
\hline \multirow{2}{*}{$\begin{array}{l}\text { Prenzler } \\
\text { et al. ( } 75)\end{array}$} & Post-OP-HV & & & & & \\
\hline & $\mathrm{HG}$ & & & & & \\
\hline \multirow{2}{*}{$\begin{array}{l}\text { Thomas } \\
\text { et al. (97) }\end{array}$} & Post-OP-HV & ns & ssssss & ns & ns & \\
\hline & $\mathrm{HG}$ & ns & ns & ns & ns & \\
\hline \multirow[t]{2}{*}{ Eichhorn } & Post-OP-HV & ns & ns & ns & ns & ns \\
\hline & $\mathrm{HG}$ & ns & ns & ns & ns & ns \\
\hline \multirow{2}{*}{$\begin{array}{l}\text { Nagai et al. } \\
(65)\end{array}$} & Post-OP HV & & & & & \\
\hline & $\mathrm{HG}$ & & & & & \\
\hline \multirow{2}{*}{$\begin{array}{l}\text { Maier et al. } \\
(57)\end{array}$} & Post-OP HV & & & & & \\
\hline & $\mathrm{HG}$ & ns & & ns & & \\
\hline \multirow{2}{*}{$\begin{array}{l}\text { Reineke et } \\
\text { al. (78) }\end{array}$} & Post-OP-HV & & & & & \\
\hline & $\mathrm{HG}$ & ns & $\mathrm{ns}$ & & & \\
\hline
\end{tabular}

\begin{tabular}{l|l|l|l|l|l|l}
\hline & & Auslöser & Schwindel & Tinnitus & IOP & Ausgangs-HV \\
\hline $\begin{array}{l}\text { Haubner } \\
\text { et al. (29) }\end{array}$ & Post-OP-HV & & ns & ns & & sssssssssss \\
\hline $\begin{array}{l}\text { Hoch et al. } \\
\text { (30) }\end{array}$ & Post-OP-HV & & & & ns & \\
\hline $\begin{array}{l}\text { Kampfner } \\
\text { et al. (33) }\end{array}$ & Post-OP-HV & & ns & ns & & \\
\hline $\begin{array}{l}\text { Prenzler et } \\
\text { al. (75) }\end{array}$ & Post-OP-HV & & & & & \\
\hline
\end{tabular}


Tab. 5.2 (Fortsetzung)

\begin{tabular}{|c|c|c|c|c|c|c|}
\hline & & Auslöser & Schwindel & Tinnitus & IOP & Ausgangs-HV \\
\hline & HG & & & & ns & \\
\hline \multirow{2}{*}{$\begin{array}{l}\text { Thomas } \\
\text { et al. (97) }\end{array}$} & Post-OP-HV & ns & sssssssssss & $\mathrm{ns}$ & $\mathrm{ns}$ & ssssssssssss \\
\hline & HG & ssssssss & sssssssssss & ns & ns & nnnnnnnn \\
\hline \multirow[t]{2}{*}{ Eichhorn } & Post-OP-HV & ns & sssssssssss & ns & ns & ssssssssssss \\
\hline & HG & ns & ns & $\mathrm{ns}$ & ns & nnnnnnnn \\
\hline \multirow{2}{*}{$\begin{array}{l}\text { Nagai } \\
\text { et al. (65) }\end{array}$} & Post-OP-HV & & & & & \\
\hline & HG & ns & ns & & & \\
\hline \multirow{2}{*}{$\begin{array}{l}\text { Maier } \\
\text { et al. (57) }\end{array}$} & Post-OP-HV & & sssssssssss & & ns & \\
\hline & HG & ns & ssssssssss & & $\mathrm{ns}$ & \\
\hline \multirow{2}{*}{$\begin{array}{l}\text { Reineke } \\
\text { et al. (78) }\end{array}$} & Post-OP-HV & & & & & ssssssssssss \\
\hline & $\mathrm{HG}$ & & $\mathrm{ns}$ & $\mathrm{ns}$ & $\mathrm{ns}$ & \\
\hline
\end{tabular}

\begin{tabular}{|c|c|c|c|c|c|c|}
\hline & & $\begin{array}{l}\text { FL-SL- } \\
\text { Mode }\end{array}$ & $\begin{array}{l}\text { Zeit } \\
\text { HS } \rightarrow \text { OP }\end{array}$ & Zeit Stat & SPN & RÜ \\
\hline \multirow{2}{*}{$\begin{array}{l}\text { Haubner } \\
\text { et al. (29) }\end{array}$} & Post-OP-HV & & & & & \\
\hline & HG & & & & & \\
\hline \multirow{2}{*}{$\begin{array}{l}\text { Hoch } \\
\text { et al. (30) }\end{array}$} & Post-OP-HV & & & & & \\
\hline & HG & ssssssssssss & sssssssssss & & & \\
\hline \multirow{2}{*}{$\begin{array}{l}\text { Kampfner } \\
\text { et al. (33) }\end{array}$} & Post-OP-HV & & & & & \\
\hline & HG & sssssssssss & Sssssssssss & & & \\
\hline \multirow{2}{*}{$\begin{array}{l}\text { Prenzler et } \\
\text { al. (75) }\end{array}$} & Post-OP-HV & & & & & \\
\hline & HG & & & & & \\
\hline \multirow{2}{*}{$\begin{array}{l}\text { Thomas } \\
\text { et al. (97) }\end{array}$} & Post-OP-HV & ns & ns & & sssssssssss & ssssssssssss \\
\hline & HG & Sssssssssss & sssssssss & & Sssssssssss & Sssssssssss \\
\hline \multirow[t]{2}{*}{ Eichhorn } & Post-OP-HV & ns & ns & ns & ns & ns \\
\hline & HG & ns & ns & ns & ns & ssssssssssss \\
\hline \multirow{2}{*}{$\begin{array}{l}\text { Nagai et al. } \\
(65)\end{array}$} & Post-OP-HV & & & & & \\
\hline & HG & & & & & \\
\hline $\begin{array}{l}\text { Maier } \\
\text { et al. (57) }\end{array}$ & Post-OP-HV & ns & & ns & & \\
\hline
\end{tabular}


Tab. 5.2 (Fortsetzung)

\begin{tabular}{l|l|l|l|l|l|l}
\hline & & $\begin{array}{l}\text { FL-SL- } \\
\text { Mode }\end{array}$ & $\begin{array}{l}\text { Zeit } \\
\text { HS } \rightarrow \text { OP }\end{array}$ & Zeit Stat & SPN & RÜ \\
\hline & HG & & & & & \\
\hline $\begin{array}{l}\text { Reineke et } \\
\text { al. (78) }\end{array}$ & Post-OP-HV & & & & & \\
\hline & HG & ns & sssssssssss & & & \\
\hline
\end{tabular}

" $n s^{\prime \prime}=$ keine signifikanten Unterschiede zwischen Zutreffen bzw. Nichtzutreffen des gesetzten Filterkriteriums; , sssssss “ = signifikante Unterschiede zwischen Zutreffen bzw. Nichtzutreffen des gesetzten Filterkriteriums; Vorerkrankung = Innenohrvorerkrankung/Schädel-Hirn-Trauma; Vorher $=$ ambulante Vorbehandlung; $I O P=$ intraoperativ pathologischer Paukenbefund; Ausgangs- $H V=$ Anfangshörverlust; $F L-S L-M o d e=$ First-Line- und Second-Line-Modus; Zeit $H \rightarrow$ $O P=$ Latenzzeit zwischen Hörsturzereignis und Tympanoskopie; $S P N=$ Spontannystagmus; $R \ddot{U}=$ Richtungsüberwiegen bei der kalorischen Reaktion; Post-OP-HV= postoperativer Hörverlust; $H G=$ Hörgewinn

eine Vorschädigung des Ohres auf den regenerativen Verlauf einer weiteren Läsion (Folgeerkrankung) negativ auswirken kann. Insofern halten wir es für sinnvoll, diesem Phänomen bei der Anamnese besondere Aufmerksamkeit zu widmen.

\subsubsection{Ambulante Vortherapien und Hörerholung}

Bezogen auf eine ambulante Vortherapie konnten wir keine statistisch relevanten Zusammenhänge zum Hörgewinn bzw. zu den Hörmessdaten in der Nachuntersuchung feststellen (Tab. 5.1). Wir fanden in der Literatur keine andere Autorengruppe, die dieser Frage bereits nachgegangen war.

\subsubsection{Für eine PLF typische Auslösesituation und Hörerholung}

Die für das Entstehen einer Perilymphfistel typischen Auslösesituationen wirkten sich in unserem Krankengut dahingehend signifikant auf die Hörentwicklung nach Mittelohroperation aus, dass Patienten, bei denen eine „elicitor situation“ bestand, schlechtere Hörlevel im Rahmen der Nachuntersuchung erreichten als Patienten, bei denen der Hörsturz ohne eine derartige Begleiterscheinung aufgetreten war $(p=0,036$; Endhörverlust mit Auslösesituation: 84,8 dB; SAW: $17,7 \mathrm{~dB} ; \mathrm{n}=11$; Endhörverlust ohne Auslösesituation: 65,4 dB; SAW: 36,3 dB; $\mathrm{n}=26$ ) (Tab. 5.1). Nach Anwendung der Bonferroni- wie auch der Li-Korrektur hielt diese Aussage aber nicht mehr dem 5-\%-Signifikanzniveau stand.

Das Vorliegen einer für das Auftreten einer PLF charakteristischen Auslösesituation hatte sich in dem Krankengut von Thomas et al. signifikant auf den Hörgewinn ausgewirkt [97]. Nagai et al. und Maier et al. konnten dies hingegen nicht bestätigen (Tab. 5.2) [57, 67]. 


\subsubsection{Schwindel und Hörerholung}

Darüber hinaus bestand in unserem Patientenkollektiv ein signifikanter Unterschied im mittleren Endhörvermögen zwischen Patienten, bei denen synchron zum Hörsturz ein Schwindel aufgetreten war, und Patienten ohne synchronen Schwindel (Endhörverlust mit Schwindel: 84,6 dB, SAW: 30,5; $\mathrm{n}=24$; Endhörverlust ohne Schwindel: 57,0 dB; SAW: 26,7 dB; $\mathrm{n}=18 ; \mathrm{p}=0,003$ ) (Tab. 5.1). Dieses Ergebnis hielt auch den Post-hoc-Adjustierungen stand ( $\mathrm{p}=0,042$ nach Bonferroni-Korrektur; $\mathrm{p}=0,02$ nach Li-Korrektur).

In den Untersuchungskollektiven von Maier et al. und Thomas et al. hatte sich das Vorhandensein von Schwindel ebenfalls signifikant negativ unterscheidend sowohl auf den Hörgewinn wie auch den im Rahmen der Nachuntersuchung dokumentierten, verbliebenen Hörverlust ausgewirkt [57, 97] (Tab. 5.2). Dies korreliert mit unseren Beobachtungen zumindest hinsichtlich des im Rahmen der Nachuntersuchung verbliebenen Resthörverlustes. Mehrere andere Autoren hingegen konnten in ihrem Krankengut keinerlei Beziehung zwischen dem Schwindel und den von den Patienten erreichten Hörgewinnen bzw. Endhörverlusten ermitteln [29, 30, 33, 65, 78].

Von allen untersuchten Parametern besteht für den synchron zum Hörsturz aufgetretenen Schwindel am ehesten eine Beziehung zum Hörsturzgeschehen, was weitere Untersuchungen noch nachzuweisen hätten.

Im Rahmen ihrer Metaanalyse sind Yu et al., allerdings unabhängig von dem gewählten Therapieverfahren in der Behandlung der ISSNHL, der Frage des Zusammenhangs zwischen dem bei einem Hörsturz synchron auftretenden Schwindel und der Hörerholung nachgegangen. Sie konnten in einem 1709 Patienten umfassenden Kollektiv einen deutlich besseren Hörgewinn bei der Gruppe jener Erkrankter feststellen, die über keinen Schwindel $(60,3 \%)$ berichtet hatten [110].

\subsubsection{Nystagmusgeschehen und Hörerholung}

\section{Hintergrundinformation}

Patienten ohne Spontannystagmus erreichten in unserem Patientengut mit einem durchschnittlichen Hörgewinn von 33,2 dB (SAW: 24,7 dB; $n=38 ; p=0,048$ ) und einem Endhörwert von 64,6 dB (SAW: 28,8 dB; $n=38 ; p=0,013$ ) bessere Resultate als jene Patienten, bei denen anfangs ein Spontannystagmus registriert werden konnte (Hörgewinn: 16,7 dB; SAW: 26,9 dB; $\mathrm{n}=15$; Endhörverlust: 90,4 dB; SAW: $29,2 \mathrm{~dB} ; \mathrm{n}=15$ ), wobei diese Aussage sich nur in einem ersten Auswertungsschritt und nur bezogen auf die Endhörwerte auf dem Signifikanzniveau sichern ließen $(\mathrm{p}=0,013)$ (Tab. 5.1 und Abb. 5.2, rechter Abbildungsteil). Der Post-hocAdjustierung hielt dieses Ergebnis auf dem 5-\%-Level nach der Bonferroni-Korrektur nicht mehr stand, wohingegen er sich nach der Li-Adjustierung bestätigte (nach Bonferroni-Korrektur $\mathrm{p}=0,18$; nach Li-Korrektur $\mathrm{p}=0,02$ ).

Zwischen Spontannystagmus (SPN) und Richtungsüberwiegen der Nystagmusantworten bestand in unserem Patientengut eine Korrelation von $\mathrm{r}=0,52$ (Abb. 5.2, linker Abbildungsteil). Dieser relativ enge Wertezusammenhang überrascht nicht, reflektieren beide Parameter doch in unterschiedlich differenzierter Form die „summarische“ Verarbeitung der aus beiden peripheren Vestibularorganen eintreffenden Nervenimpulse im Stammhirnbereich. 


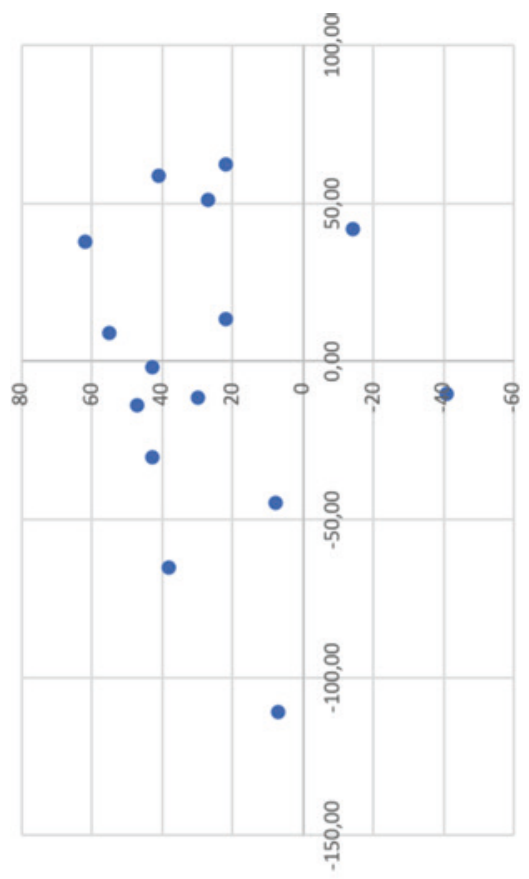

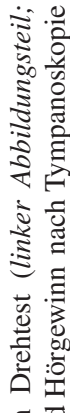

$\Xi \Xi$

気云

気

है

20

栗鱼

$z, \Xi$

式 芯

웡 긍

$0_{0.0}$

: $:$

폰

.

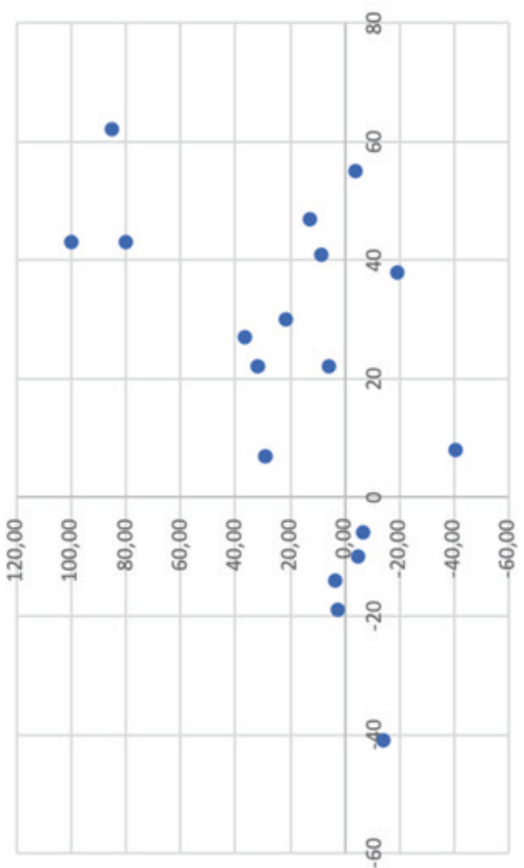

䓃寻

,

ఫ识

三 至

乙这

氙

苛

हैं :

5o

is

色苍

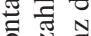

은

की $\frac{\text { व }}{5}$

区 थ

$\ddot{U} . \Xi$

z.

0000

路

ए

ఏ)

곤호

ก้

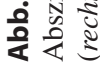


Gliedert man die Patienten in zwei Gruppen, wobei die einen ein Richtungsüberwiegen von $>25 \%$, die andere von $<25 \%$ aufwiesen, so boten jene mit einem ausgeprägten rotatorischen Richtungsüberwiegen einen Hörgewinn von $35 \mathrm{~dB}$ (SAW: $34,5 \mathrm{~dB} ; \mathrm{n}=7$ ) bei einem Endhörverlust von $74 \mathrm{~dB}$ (SAW: 48,3 dB; n=7), wohingegen Patienten mit geringerer „directional preponderance“ nur einen Hörgewinn von 16,4 dB (SAW: 24,6 dB; $n=11$ ) bei einem langfristig gemessenen Hörwert von 91 dB (SAW: 19,3 dB; $n=11$ ) erreichten.

In den Arbeiten von Thomas et al. zeigte sich, dass nicht nur der Schwindel, sondern auch das objektivierbare Nystagmusgeschehen, ersichtlich am Spontannystagmus, zum mittleren Endtonhörverlust in einer statistisch signifikanten Beziehung steht (Tab. 5.2) [97]. Für die Kalorisation hingegen konnten weder Thomas et al. noch wir einen signifikanten Zusammenhang zum Symptom Schwindel herstellen [97]. Im Gegensatz zu unseren Beobachtungen zeigt dieses Ergebnis, dass bei dem Versuch, die Schwindelbeschwerden zu objektivieren und ihre Herkunft in den Vestibularapparat zu lokalisieren, der Zusammenhang zwischen den Gleichgewichts- und Innenohrhörstörungen weniger eindeutig hergestellt werden konnte.

\subsubsection{Tinnitus und Hörerholung}

\section{Hintergrundinformation}

Auch das gleichzeitige Auftreten von Tinnitus erwies sich in unsrem Patientenkollektiv als nicht signifikant prognoserelevant bezogen auf den Hörgewinn bzw. die Höhe des langfristig verbleibenden Hörverlustes (Tab. 5.1).

Zu gleichgearteten Ergebnissen kamen verschiedene Autorengruppen, die dieser Frage ebenfalls nachgegangen waren (Tab. 5.2) [29, 30, 75, 97].

\subsubsection{Intraoperativ pathologischer Paukenbefund und Hörerholung}

Weder in unserem Patientenkollektiv noch in anderen Studiengruppen zeigte sich ein statistisch signifikanter Unterschied in Bezug auf die Hörerholung zwischen der Untergruppe der Patienten mit Hörsturz, die bei der explorativen Tympanoskopie auffällige Paukenstrukturen (in der Regel im Sinne einer Perilymphfistel) hatten erkennen lassen, und jenen, bei denen regelechte Mittelstrukturen bestanden (Tab. 5.1 und 5.2) [29, 57, 75, 78, 97].

\subsubsection{Latenzzeit zwischen Hörsturzereignis und Tympanoskopie sowie Hörerholung}

Die Latenzzeit, die zwischen dem Hörsturzereignis und dem Termin der Tympanoskopie lag, beeinflußte in unserem Patientenkollektiv das Ausmaß des Hörgewinns und den letztendlich erreichten, langfristigen mittleren Hörverlust nicht signifikant (Tab. 5.1 und Abb. 5.1). Auch die Dauer der präoperativen Behandlung mit systemischen Kortikoidinfusionen vor Durchführung der 
Tympanoskopie zeigte keine signifikanten Auswirkungen auf das Ausmaß der postoperativ einsetzenden Hörerholung bzw. der Höhe des im Rahmen der Nachuntersuchung gemessenen Hörlevels.

In drei aus der Literatur zusammengetragenen Studien hingegen zeigte sich ein signifikanter Einfluss der Latenzzeit, die zwischen dem Hörsturzereignis und der Tympanoskopie vergangen war, auf die weitere Hörentwicklung, derart, dass eine verzögert durchgeführte Operation mit geringeren Hörerwartungen einherging als ein frühzeitiger chirurgischer Eingriff [29, 33, 78]. Insofern muss zumindest damit gerechnet werden, dass sich eine später angesetzte Operation auch nachteilig auf den Hörerfolg auswirken kann.

\subsubsection{Initialer Hörverlust und Hörerholung}

Bei den von uns untersuchten Erkrankten betrug der langfristige Hörverlust bei denjenigen, die anfangs eine durchschnittliche Hörminderung von $>100 \mathrm{~dB}$ aufgewiesen hatten noch 85,9 dB, (SAW: 24,6 dB; $n=21$ ), bei Patienten mit initial $<100 \mathrm{~dB}$ Hörminderung lagen die Endhörwerte bei 60,8 dB (SAW: 32,8 dB; $\mathrm{n}=19$ ) (Tab. 5.2 und Abb. 5.1). Dieser Unterschied erwies sich statistisch auch nach Anwendung der Bonferroni- $(\mathrm{p}=0,014)$ und Li-Korrektur $(\mathrm{p}=0,006)$ als signifikant.

$\mathrm{Zu}$ vergleichbaren Ergebnissen waren auch Haubner et al., Prenzler et al., Reineke et al. und Thomas et al. gekommen [29, 75, 78, 97]. Für das Ausmaß der Hörgewinne konnte hingegen keine der angeführten Studiengruppen eine signifikante Beziehung zur Höhe des Ausgangshörverlustes ermitteln. Diese Beobachtung legt nahe, dass mit der Tympanoskopie inkl. Abdichtung der runden/ ovalen Fensternischen in Verbindung mit einer systemischen Kortikoidbehandlung ein positiver Behandlungseffekt erreicht werden kann.

Interessant erscheint die Beobachtung von Reineke et al. und Ulk-Mulk et al., die auch Patienten mit mittleren Hörverlusten $<60 \mathrm{~dB}$ in ihrem Untersuchungsgut aufgenommen hatten (gewichteter Mittelwert: 60,8 dB), dass diese von ihnen behandelten Patienten einen gewichteten mittleren Hörgewinn von 12,7 dB erlangen konnten (Vergleichswert der Autoren mit Ausgangshörverlusten > $60 \mathrm{~dB}$ : 32,7 dB) [29, 30, 33, 78, 97, 101]. Als Endhörverluste dokumentierten Reineke et al. sowie Ulk-Mulk et al. zusammengefasst einen gewichteten Mittelwert von 48,1 dB, dem eine verbleibende Höreinschränkung von 68,0 dB in der Gruppe der Publikationen gegenüberstand, bei denen die anfänglichen Höreinschränkungen der Patienten ausnahmslos $>60 \mathrm{~dB}$ betrugen ([29, 30, 33, 97] und eigene Studie). Dies lässt die Vermutung aufkommen, dass bei geringeren Ausgangshörverlusten nicht mehr so hohe Hörgewinne erreicht werden können, wie dies bei ausgeprägteren Ausgangshörverlusten der Fall ist. Diese Beobachtung kann v. a. dann eine Bedeutung erlangen, wenn die Hörergebnisse der tympanoskierten Patienten mit ausschließlich hochgradigen initialen Hörminderungen/Taubheiten mit den Hörresultaten anderer, alternativ eingesetzter Therapieverfahren verglichen werden, bei denen auch Erkrankte mit weniger starken Anfangshörverlusten in eine Auswertung einbezogen wurden. 


\subsubsection{Hörkurventyp und Hörerholung}

Ordnet man die Patienten unseres Kollektives mit ihren Ausgangshörverlusten einzelnen Hörkurvenverlaufstypen $\mathrm{zu}$, so fanden wir 9-mal $(24,4 \%)$ einen apicikochleären, 1-mal (2,4\%) einen zeltförmigen, 4-mal (9,5\%) einen basokochleären und 28-mal $(66,7 \%)$ einen pankochleären Schwerhörigkeitstyp. Letztere Gruppe setzt sich allein aus 13 Erkrankten $(31,0 \%)$ zusammen, die eine komplette Taubheit aufwiesen. Die Patienten mit auffälligen pathoanatomischen Mittelohrbefunden wiesen ein gemischtes Bild auf, wobei 1 apikokochleärer, 1 basokochleärer und 2 pankochleäre Schwerhörigkeitstypen zur Beobachtung kamen.

Bei den Patienten mit pankochleärer Schwerhörigkeit, die auch die Gruppe der Ertaubten einschloss, verbesserte sich der gemittelte Hörverlust von anfänglich 106,3 dB um 32,9 dB auf den Endwert von 73,4 dB (Abb. 5.3). Betrachtet man lediglich die Erkrankten mit pankochleärer Schwerhörigkeit, die nicht ertaubt waren, konnte man einen Anstieg des mittleren Hörverlustes von 95,1 dB ausgehend um 39,8 dB auf einen Hörwert im Rahmen der Nachuntersuchung von 55,3 dB beobachten. Die Patienten mit apicikochleärer Hörminderung steigerten ihr Hörvermögen von durchschnittlich $92,6 \mathrm{~dB}$ um 22,3 dB auf 70,3 dB. In der Gruppe der Erkrankten mit einem basokochleären Schwerhörigkeitstyp entwickelte sich das Hörvermögen von initial 88,7 dB im Mittel um 31,2 dB auf den Endwert von $57,5 \mathrm{~dB}$. Mit einem $\mathrm{p}=0,29$ ließ sich im Rahmen der Varianzanalyse (ANOVA) kein statistisch signifikanter Unterschied zwischen den einzelnen Gruppen ermitteln.

Insgesamt zeigten also die pankochleär von der Schwerhörigkeit befallenen Patienten, unabhängig davon, ob Ertaubte mit eingeschlossen waren oder nicht, die besten prognostischen Erwartungen. Am Ende bot sich, mehr oder weniger unabhängig vom Ausgangsbefund, das Bild einer zu den hohen Tönen hin abfallenden Hörkurve. Vergleichbare Erhebungen sind aus der Literatur nicht bekannt.

\subsubsection{ANOVA}

Bei der Durchführung der Varianzanalyse (ANOVA) ergab sich, dass sich keine der aus unserem Gesamtkollektiv gebildeten Subpopulationen hinsichtlich des Hörgewinns $(p=0,67)$ wie auch des Endhörverlustes $(p=0,27)$ signifikant von einer anderen unterschied.

\subsubsection{Abdeckung des runden und/oder ovalen Fensters}

Zur Frage, ob es im Heilungsverlauf einen Unterschied gibt, ob das runde und/ oder ovale Fenster gleichzeitig mit autologem Bindgewebe abgedichtet werden, kann aus eigener Erfahrung kein Beitrag geliefert werden, da wir immer beide Fensternischen obliteriert haben. Bislang haben sich mit dieser Thematik ausschließlich Hofmann et al. [31] beschäftigt. Von statistisch sich nicht unterscheidenden Ausgangshörwerten, konnten sie direkt nach der Tamponade bei den Patienten mit Abdichtung beider Fenster einen durchschnittlichen medianen 

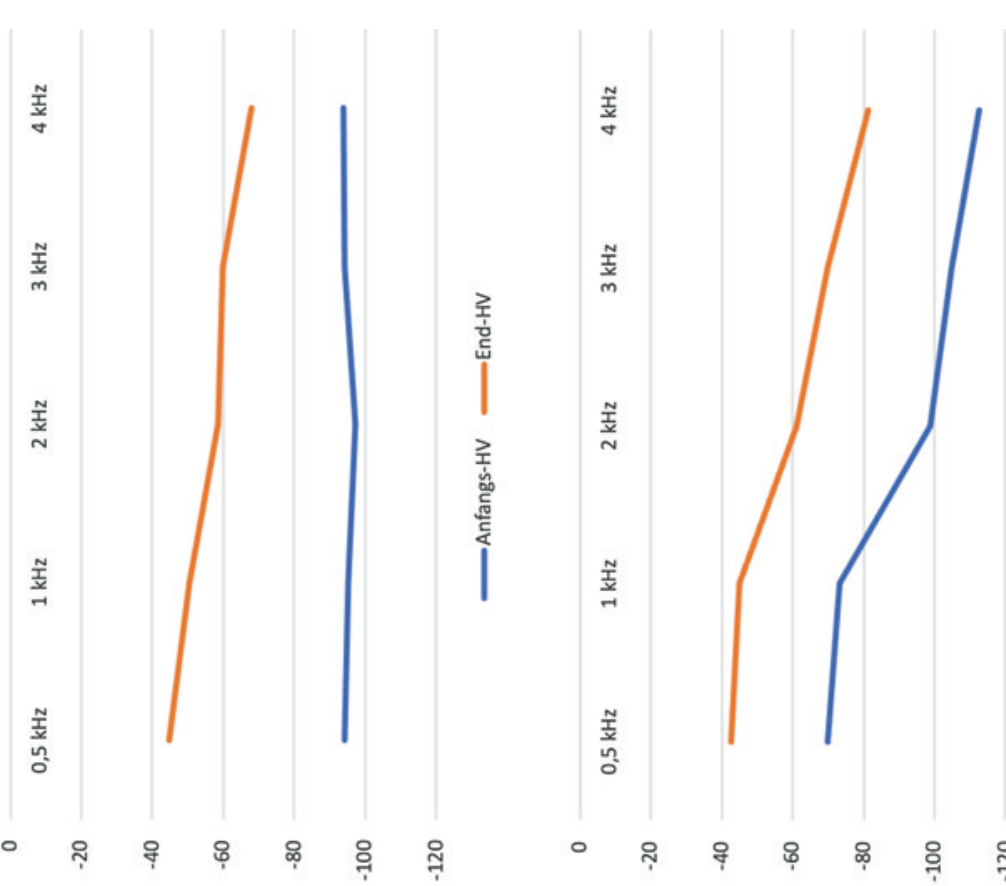

离

늄

茥

๖.

등

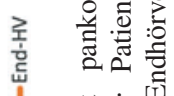

当荄可

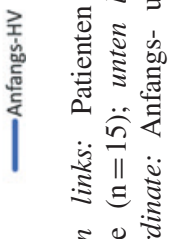

ธิ

志 $\dot{\square}$

包牙 I

害 き

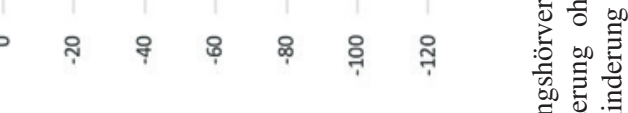

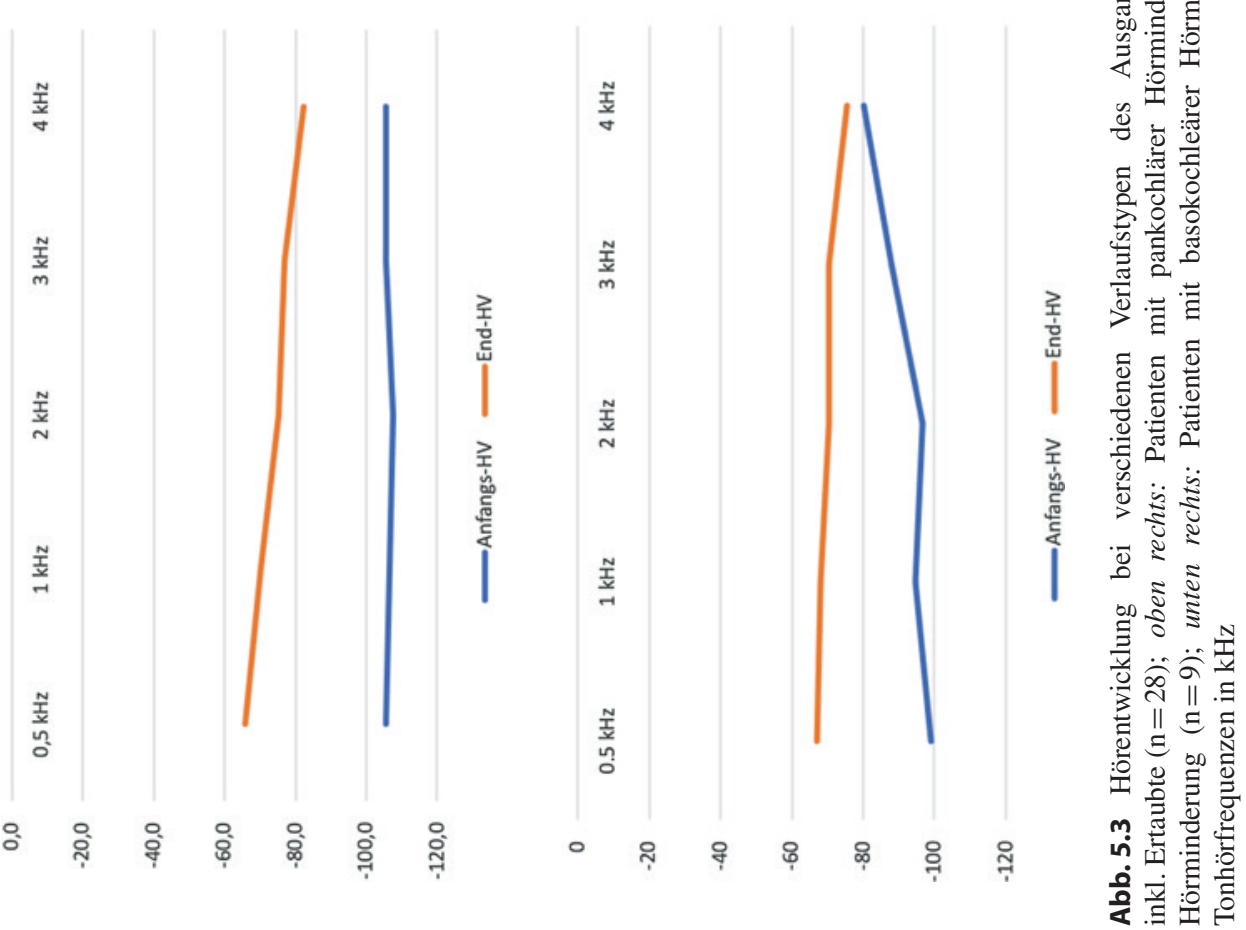


Tab. 5.3 Hörverbesserungen nach Tympanoskopie, gemessen anhand der Kanzaki-Kriterien [35]

\title{
Hörverbesserungen nach „Kanzaki“-Kriterien
}

\author{
$>30 \mathrm{~dB}$ \\ („complete“ und „marked“) \\ Eigene Studie $\quad 45,20 \%$ \\ Thomas et al. $\quad 64,80 \%$ \\ Hoch et al. $\quad 62,70 \%$
}

Hörgewinn von jeweils $15 \mathrm{~dB}$ beobachten. Langfristig war es in der Gruppe der Erkrankten, bei denen sowohl die ovale wie auch die Rundfenstermembran obliteriert worden waren, zu einer weiteren Hörverbesserung von $13,1 \mathrm{~dB}$ vs. 7,5 dB gekommen, wenn nur die runde Fensternische abgedichtet worden war. Beide Werte boten keinen signifikanten Unterschied.

\subsubsection{Hörerholung nach den modifizierten Kanzaki-Kriterien}

Legt man die Kanzaki-Kriterien bei der Auswertung der Hörerholungen zugrunde, so zeigte sich die höchste Anzahl der Hörbesserungen mit $>30 \mathrm{~dB}$ (Typ 1 und Typ 2) bei Thomas et al. (64,8\%) (Tab. 5.3) [97]. Unser Patientenkollektiv rangierte demgegenüber mit einem Hörgewinn der Typen 1 und 2 von 45,2\% im Vergleich zu den anderen Studien am unteren Ende der Skala [30, 97].

Die Kanzaki-Kriterien eignen sich unserer Meinung nach für die Bewertung des Hörgewinn der von uns untersuchten Patientenkollektive weitaus besser als die sog. Siegel-Kriterien, die sich am Ausmaß des Endhörverlustes orientieren. Bei unserem Patientenkollektiv mit ausschließlich hochgradigen anfänglichen Hörminderungen/Taubheiten werden zum Teil zwar erhebliche Hörgewinne erreicht, dennoch weisen die Endhörverluste nicht den Bereich auf, der nach den Siegel-Kriterien einen ausreichenden Erfolg attestiert hätte. Insofern glauben wir, dass die alleinige Orientierung an den Hörgewinnen bei den tympanoskopierten Patienten, auch wenn sie im Vergleich mit andersartig behandelten Patientengruppen verglichen werden, am meisten Sinn ergibt. 


\subsection{Zusammenfassung}

Bei der explorative Tympanoskopie mit Abdichtung der runden/ovalen Fensternischen in der Behandlung der fortgeschrittenen ISSNHL zeigt sich - wie bei anderen Behandlungsverfahren dieser Erkrankung auch - dass diese Therapieform bei einigen Erkrankten mit größeren, bei anderen mit geringeren prognostischen Erwartungen einhergeht. In diesem Kapitel wird der Frage nachgegangen, welche patientenassoziierten Merkmale sich statistisch signifikant auf den Heilungserfolg auswirken.

Anhand eines Kollektives, das sich aus 42 Patienten einer eigenen Untersuchung und den Daten aus sieben anderen Studien zusammensetzt $(n=539)$, die ebenfalls die explorative Tympanoskopie angewendet haben, wird anhand statistischer Verfahren verglichen, welche Parameter sich in signifikanter Weise auf die Hörentwicklung nach dem o. g. Mittelohreingriff ausgewirkt haben.

Verschiedene Parameter, wie Geschlechtszugehörigkeit, Seitenlokalisation des Hörsturzes, Vorerkrankung des betroffenen Innenohrs oder früheres SHT, ambulante orale Vortherapie, PLF-typische Auslösesituation zum Zeitpunkt des Hörsturzes wie auch ein synchron zum Hörsturz aufgetretener Tinnitus, zeigten keinen statistisch signifikanten Einfluss auf die Hörentwicklung nach dem chirurgischen Eingriff mit Abdeckung beider Fensternischen. In einer Studie aus der Literatur stellte sich das Ausmaß des Hörgewinns in Abhängigkeit vom Patientenalter dar.

Von vier Studien, die die Auswirkung der für das Entstehen einer Perilymphfistel typischen Auslösesituationen untersuchten, konnte nur eine einen signifikanten Einfluss auf den postoperativen Hörgewinn finden. In unserer Studie ergab sich ebenfalls kein statistischer Zusammenhang zwischen diesen beiden Parametern.

Mehrere Publikationen und auch unsere Studie gehen davon aus, dass sich ein zeitgleich mit dem Hörsturz aufgetretener Schwindel statistisch relevant negativ auf den Hörgewinn und auch das langfristige Hörvermögen auswirkt. Bei einem nachgewiesenen Spontannystagmus, wie auch dem aus den Nystagmusantworten ermittelten Richtungsüberwiegen, ergab sich demgegenüber ein geringerer Zusammenhang zur postoperativen Hörentwicklung.

Die mehr als 1 Monat nach der Operation gemessenen Hörlevel fielen bei mehreren Untersuchungen signifikant schlechter aus, je ausgedehnter der initiale Hörverlust war. Dieses Ergebnis fand sich auch in unserem Patientenkollektiv, indem der Hörgewinn in der Patientengruppe mit höheren Ausgangshörverlusten signifikant schlechter ausfiel als bei Patienten mit geringeren initialen Hörbeeinträchtigungen.

Die Latenzzeit, die zwischen dem Hörsturzereignis und der Tympanoskopie lag, nahm nur bei drei von sechs Studien, die dieser Themenstellung nachgegangen waren, einen signifikanten Einfluss auf den Hörgewinn.

Patienten mit pankochleären Hörkurvenverläufen im initialen Tonschwellenaudiogramm zeigten bessere durchschnittliche Hörerholungsraten 
als Erkrankte mit apico- oder basokochleären Ausgangshörminderungen, dies war jedoch statistisch nicht als signifikant verifizierbar.

Die Frage nach der Prognoserelevanz einzelner Parameter bei der Therapie des Hörsturzes mit der explorativen Tympanoskopie und Obliteration der runden/ ovalen Fensternische mit autologem Bindegewebe wird in der Literatur uneinheitlich beantwortet. Die prognostischen Erwartungen an einen Hörgewinn sind am ehesten vom Ausmaß des anfänglichen Hörverlustes und dem begleitend zur Hörminderung eingetretenen Schwindel abhängig.

Open Access Dieses Kapitel wird unter der Creative Commons Namensnennung 4.0 International Lizenz (http://creativecommons.org/licenses/by/4.0/deed.de) veröffentlicht, welche die Nutzung, Vervielfältigung, Bearbeitung, Verbreitung und Wiedergabe in jeglichem Medium und Format erlaubt, sofern Sie den/die ursprünglichen Autor(en) und die Quelle ordnungsgemäß nennen, einen Link zur Creative Commons Lizenz beifügen und angeben, ob Änderungen vorgenommen wurden.

Die in diesem Kapitel enthaltenen Bilder und sonstiges Drittmaterial unterliegen ebenfalls der genannten Creative Commons Lizenz, sofern sich aus der Abbildungslegende nichts anderes ergibt. Sofern das betreffende Material nicht unter der genannten Creative Commons Lizenz steht und die betreffende Handlung nicht nach gesetzlichen Vorschriften erlaubt ist, ist für die oben aufgeführten Weiterverwendungen des Materials die Einwilligung des jeweiligen Rechteinhabers einzuholen.

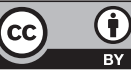

